\title{
Sensitivity on the electromagnetic dipole moments of the tau-lepton at the CLIC
}

\author{
Maria de los Ángeles Hernández-Ruíz ${ }^{1 \star}$, Ahmet A. Billur², \\ Murat Köksal $^{3}$ and Alejandro Gutiérrez-Rodríguez ${ }^{4}$
}

1 Unidad Académica de Ciencias Químicas, Universidad Autónoma de Zacatecas, Apartado Postal C-585, 98060 Zacatecas, México.

2 Deparment of Physics, Cumhuriyet University, 58140, Sivas, Turkey.

3 Deparment of Optical Engineering, Cumhuriyet University, 58140, Sivas, Turkey.

4 Unidad Académica de Física, Universidad Autónoma de Zacatecas, Apartado Postal C-580, 98060 Zacatecas, México.

$\star$ mahernan@uaz.edu.mx

Proceedings for the 15th International Workshop on Tau Lepton Physics, Amsterdam, The Netherlands, 24-28 September 2018

doi:10.21468/SciPostPhysProc.1

\begin{abstract}
In this paper we established model independent bounds on the anomalous magnetic and electric dipole moments of the tau-lepton using the process $\gamma \gamma \rightarrow \tau^{+} \tau^{-}$. We use data collected with the future $\mathrm{e}^{+} \mathrm{e}^{-}$linear collider such as the CLIC at $\sqrt{\mathrm{s}}=380,1500$, $3000 \mathrm{GeV}$, and we consider systematic uncertainties of $\delta_{\text {sys }}=0 \%, 3 \%, 5 \%$. The theory predictions are a very good prospect for probing the dipole moments of the tau-lepton at the future $\mathrm{e}^{+} \mathrm{e}^{-}$linear collider at the $\gamma \gamma$ mode.
\end{abstract}

(c) (1) Copyright M. A. Hernández-Ruíz et al. This work is licensed under the Creative Commons Attribution 4.0 International License. Published by the SciPost Foundation.
Received 19-11-2018

Accepted 10-01-2019

Published 22-02-2019

doi:10.21468/SciPostPhysProc.1.045

\section{Introduction}

In this work, using $\gamma \gamma \rightarrow \tau^{+} \tau^{-}$reaction we establish model independent sensitivity estimates on the dipole moments $a_{\tau}$ and $d_{\tau}$ of the tau-lepton. The high center-of-mass energies what has been proposed for the Compact Linear Collider (CLIC) make it an appropriate machine to probe the anomalous magnetic (MM) and electric dipole (EDM) moments which are more sensitive to the high energy and high luminosity of the collider. The CLIC is a proposed future $e^{+} e^{-}$collider, designed to fulfill $e^{+} e^{-}$collision at center-of-mass energies of $0.35 \mathrm{TeV}, 1.4 \mathrm{TeV}$ and $3 \mathrm{TeV}$ planned to be constructed with a three main stage research region. This enables the investigation of de $\gamma \gamma$ and $e \gamma$ interactions by converting the original $e^{-}$or $e^{+}$beam into a photon beam through the Compton back-scattering mechanism.

For our study we consider the following parameters of the CLIC: $\sqrt{s}=380,1500,3000$ $\mathrm{GeV}, \mathcal{L}=10,50,100,300,500,1000,1500,2000,3000 \mathrm{fb}^{-1}$, with systematic uncertainties 


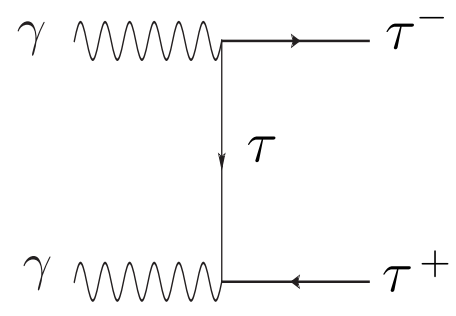

(1)

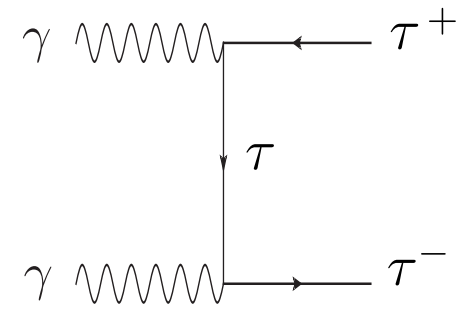

(2)

Figure 1: The Feynman diagrams for the process $\gamma \gamma \rightarrow \tau^{+} \tau^{-}$.

of $\delta_{\text {sys }}=0 \%, 3 \%, 5 \%$. We obtain strong sensitivity in comparison to the bounds given by the DELPHI, L3, OPAL, BELLE, and ARGUS Collaborations [1-5].

This paper is organized as follows: In Section 2, we present the total cross section and the electromagnetic dipole moments of the tau-lepton for the $\gamma \gamma \rightarrow \tau^{+} \tau^{-}$reaction. In section 3 , the results. In section 4, we give our conclusion.

\section{The process $\gamma \gamma \rightarrow \tau^{+} \tau^{-}$}

To calculate the $\gamma \gamma \rightarrow \tau^{+} \tau^{-}$total cross section, the corresponding Feynman diagrams are given in Fig. 1. We determine sensitivity estimates on the electromagnetic dipole moments of the tau-lepton $a_{\tau}$ and $d_{\tau}$ via the two-photon process [6]. The future Collider CLIC can produce very hard photons at high luminosity in Compton backscattering of laser light off high energy $e^{+} e^{-}$beams.

The electromagnetic current between on-shell tau-lepton and the photon is given by [7-10]

$$
\begin{aligned}
\Gamma_{\tau}^{\alpha}= & e F_{1}\left(q^{2}\right) \gamma^{\alpha}+\frac{i e}{2 m_{\tau}} F_{2}\left(q^{2}\right) \sigma^{\alpha \mu} q_{\mu}+\frac{e}{2 m_{\tau}} F_{3}\left(q^{2}\right) \sigma^{\alpha \mu} q_{\mu} \gamma_{5} \\
& +e F_{4}\left(q^{2}\right) \gamma_{5}\left(\gamma^{\alpha}-\frac{2 q^{\alpha} m_{\tau}}{q^{2}}\right),
\end{aligned}
$$

where the $q^{2}$-dependent form factors $F_{1,2,3,4}\left(q^{2}\right)$ have interpretations for $q^{2}=0: F_{1}(0)=Q_{\tau}$ is the electric charge; $F_{2}(0)=a_{\tau}$ is anomalous MM and $F_{3}(0)=\frac{2 m_{\tau}}{e} d_{\tau}$ with $d_{\tau}$ the EDM. $F_{4}\left(q^{2}\right)$ is the anapole form factor. Here, $e$ is the charge of the electron, $m_{\tau}$ is the mass of the tau-lepton, $\sigma^{\alpha \mu}=\frac{\mathrm{i}}{2}\left[\gamma^{\alpha}, \gamma^{\mu}\right]$ represents the spin $1 / 2$ angular momentum tensor, and $q=p^{\prime}-p$ is the momentum transfer.

The spectrum of Compton backscattered photons to the process $\gamma \gamma \rightarrow \tau^{+} \tau^{-}$is given by

$$
f_{\gamma}(y)=\frac{1}{g(\zeta)}\left[1-y+\frac{1}{1-y}-\frac{4 y}{\zeta(1-y)}+\frac{4 y^{2}}{\zeta^{2}(1-y)^{2}}\right]
$$

where

$$
g(\zeta)=\left(1-\frac{4}{\zeta}-\frac{8}{\zeta^{2}}\right) \log (\zeta+1)+\frac{1}{2}+\frac{8}{\zeta}-\frac{1}{2(\zeta+1)^{2}}
$$

and

$$
y=\frac{E_{\gamma}}{E_{e}}, \quad \zeta=\frac{4 E_{0} E_{e}}{M_{e}^{2}}
$$




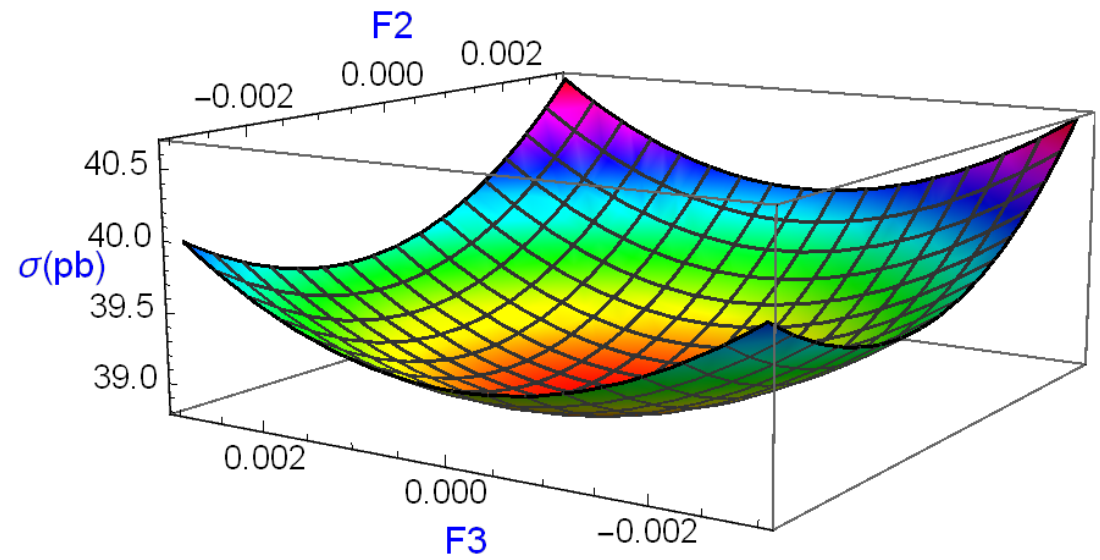

Figure 2: The total cross sections of the process $\gamma \gamma \rightarrow \tau^{+} \tau^{-}$as a function of $F_{2}$ and $F_{3}$ for center-of-mass energy of $\sqrt{s}=380 \mathrm{GeV}$.

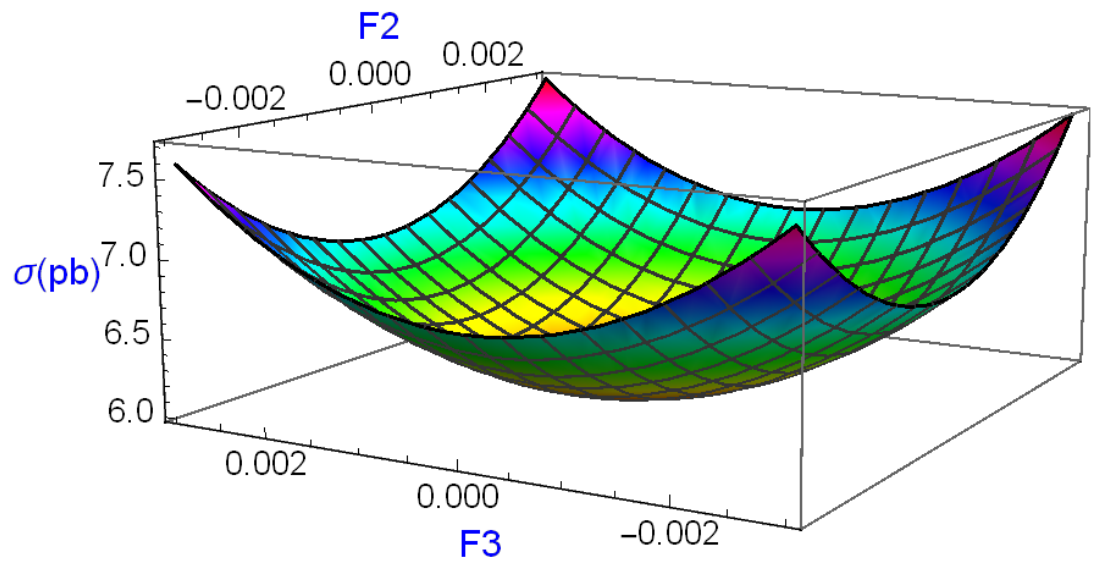

Figure 3: The same as in Figure 2, now for $\sqrt{s}=1500 \mathrm{GeV}$.

$E_{0}$ is energy of the incoming laser photon while for $E_{e}$ is initial energy of the electron beam before Compton backscattering, and $E_{\gamma}$ is the energy of the backscattered photon.

The total cross section can be written as,

$$
\sigma=\int f_{\gamma}(x) f_{\gamma}(x) d \hat{\sigma} d E_{1} d E_{2}
$$

where $E_{1}$ and $E_{2}$ is the energy of the particles of the final state. 
Now, we present the total cross section as a polynomial in powers of $F_{2}$ and $F_{3}$ for the process $\gamma \gamma \rightarrow \tau^{+} \tau^{-}$. The formulas have been obtained with the help of the package CALCHEP [11], which can computate the Feynman diagrams, integrate over multiparticle face space and event simulation.

- For $\sqrt{s}=380 \mathrm{GeV}$.

\section{Results}

$$
\begin{aligned}
& \sigma\left(F_{2}\right)=\left[(9914034) F_{2}^{4}+(81889) F_{2}^{3}+(81382) F_{2}^{2}+(111) F_{2}+38.75\right](p b) \\
& \sigma\left(F_{3}\right)=\left[(9736246) F_{3}^{4}+(82619) F_{3}^{2}+38.75\right](p b)
\end{aligned}
$$

- For $\sqrt{s}=1500 \mathrm{GeV}$.

$$
\begin{aligned}
& \sigma\left(F_{2}\right)=\left[\left(1.54 \times 10^{8}\right) F_{2}^{4}+(84288) F_{2}^{3}+(88058) F_{2}^{2}+(17.5) F_{2}+6\right](p b), \\
& \sigma\left(F_{3}\right)=\left[\left(1.54 \times 10^{8}\right) F_{3}^{4}+(88124) F_{3}^{2}+6\right](p b) .
\end{aligned}
$$

- For $\sqrt{s}=3000 \mathrm{GeV}$.

$$
\begin{aligned}
& \sigma\left(F_{2}\right)=\left[\left(6.17 \times 10^{8}\right) F_{2}^{4}+(91348) F_{2}^{3}+(87216) F_{2}^{2}-(1.21) F_{2}+1.97\right](p b) \\
& \sigma\left(F_{3}\right)=\left[\left(6.17 \times 10^{8}\right) F_{3}^{4}+(88327) F_{3}^{2}+1.97\right](p b)
\end{aligned}
$$

\section{Bounds on the $a_{\tau}$ and $d_{\tau}$ through $\gamma \gamma \rightarrow \tau^{+} \tau^{-}$at the CLIC}

We now proceed with our numerical analysis of the total cross section

$$
\sigma_{N P}\left(\gamma \gamma \rightarrow \tau^{+} \tau^{-}\right)=\sigma_{N P}\left(\sqrt{s}, F_{2}, F_{3}\right)
$$

as well as of the electromagnetic dipole moments of the tau-lepton, here the free parameters are $\sqrt{s}, \mathcal{L}, F_{2}$ and $F_{3}$. For this purpose, we use the usual formula for the $\chi^{2}$ function [12-15]:

$$
\chi^{2}=\left(\frac{\sigma_{S M}-\sigma_{N P}\left(\sqrt{s}, F_{2}, F_{3}\right)}{\sigma_{S M} \delta}\right)^{2},
$$

$\sigma_{N P}\left(\sqrt{s}, F_{2}, F_{3}\right)$ is the total cross section which includes contributions to the SM and new physics, $\delta=\sqrt{\left(\delta_{s t}\right)^{2}+\left(\delta_{s y s}\right)^{2}}, \delta_{s t}=\frac{1}{\sqrt{N_{S M}}}$ is the statistical error, $\delta_{s y s}$ is the systematic error and $N_{S M}$ is the number of signal expected events $N_{S M}=\mathcal{L}_{i n t} \times B R \times \sigma_{S M}, \mathcal{L}_{i n t}$ is the integrated CLIC luminosity.

In this section we presented a set of figures, which illustrate our results. The total cross sections $\sigma_{\gamma \gamma \rightarrow \tau^{+} \tau^{-}}\left(\sqrt{s}, F_{2}, F_{3}\right)$ are calculated as a function of the anomalous couplings $F_{2}$ and $F_{3}$ with the center-of-mass energies of $\sqrt{s}=380 \mathrm{GeV}, \sqrt{s}=1500 \mathrm{GeV}$ and $\sqrt{s}=3000 \mathrm{GeV}$. 


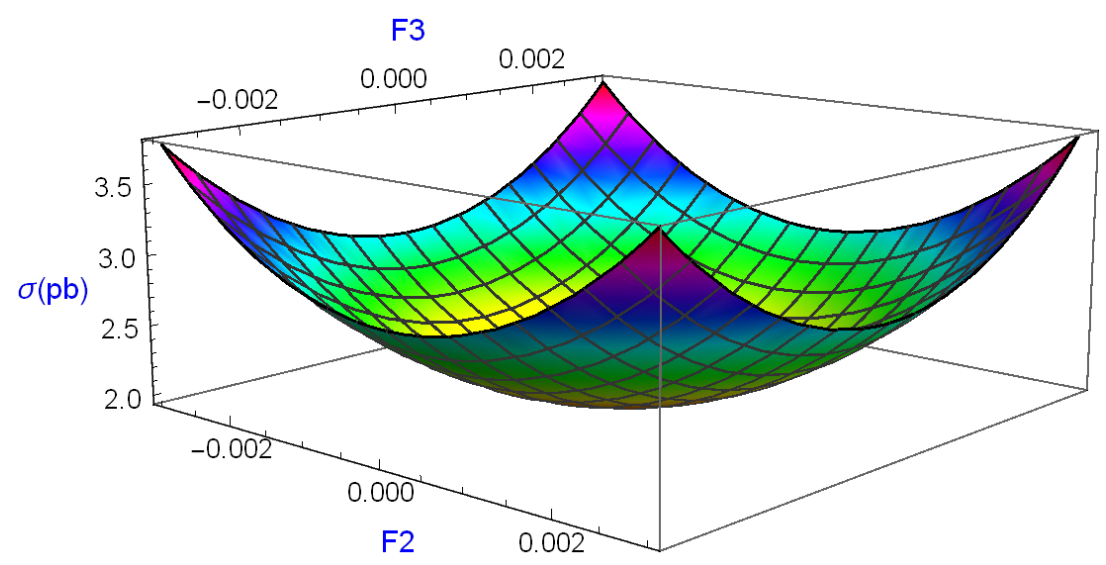

Figure 4: The same as in Figure 3, now for $\sqrt{s}=3000 \mathrm{GeV}$.

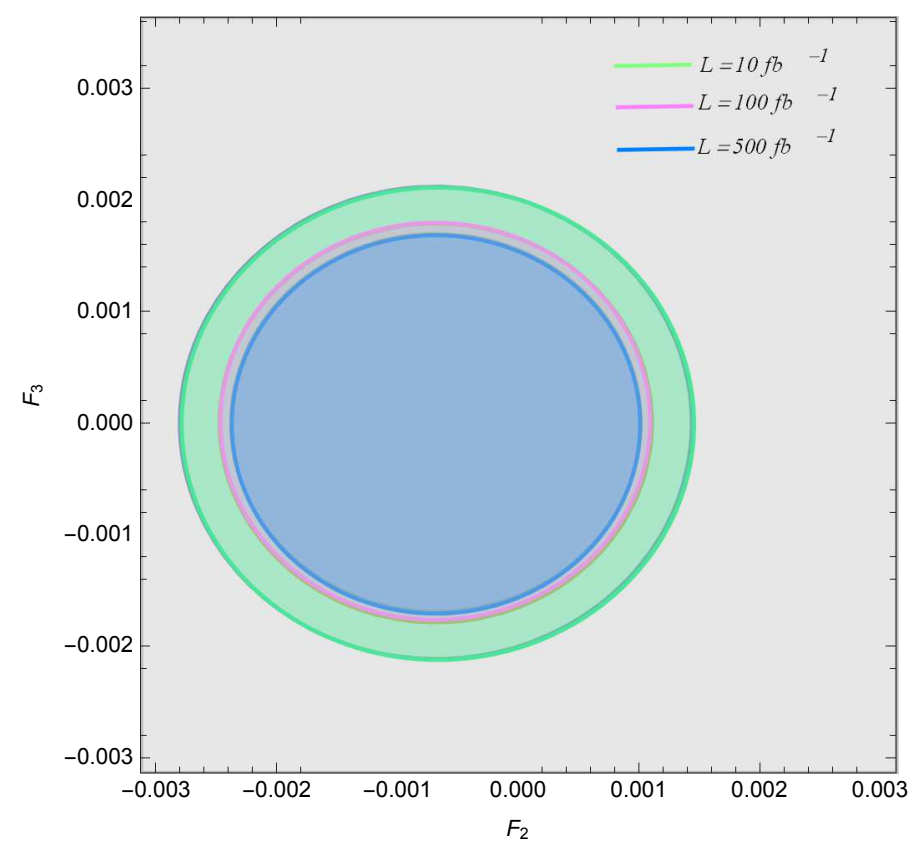

Figure 5: Bounds contours at the 95\% C.L. in the $\left(F_{2}-F_{3}\right)$ plane for the process $\gamma \gamma \rightarrow \tau^{+} \tau^{-}$with the $\mathcal{L}=10,100,500 \mathrm{bf}^{-1}$ and for center-of-mass energy of $\sqrt{s}=380 \mathrm{GeV}$.

The total cross section shows a strong dependence on the anomalous paramaters $F_{2}, F_{3}$, and the center-of-mass energy of the collider $\sqrt{s}$ as they are show in Figures 2-4.

Figures 5-7 indicate allowed regions at 95\% C.L. in the plane $\left(F_{2}-F_{3}\right)$ for the process $\gamma \gamma \rightarrow \tau^{+} \tau^{-}$during the first, second and third stage of operation of the CLIC, where assumed fixed center-of-mass energies are $\sqrt{s}=380 \mathrm{GeV}, \sqrt{s}=1500 \mathrm{GeV}$, and $\sqrt{s}=3000$ $\mathrm{GeV}$ with luminosities $\mathcal{L}=10 \mathrm{fb}^{-1}, \mathcal{L}=100 \mathrm{fb}^{-1}$, and $\mathcal{L}=500 \mathrm{fb}^{-1}$, in Figure 5; likewise $\mathcal{L}=100 \mathrm{fb}^{-1}, \mathcal{L}=500 \mathrm{fb}^{-1}$, and $\mathcal{L}=1500 \mathrm{fb}^{-1}$ in Figure 6; while, $\mathcal{L}=100 \mathrm{fb}^{-1}$, $\mathcal{L}=500 \mathrm{f} \mathrm{b}^{-1}, \mathcal{L}=3000 \mathrm{fb}^{-1}$ in Figure 7, and systematic uncertainties of $\delta_{\text {sys }}=0 \%, 3 \%, 5 \%$ $[1,16]$.

These results that we get for the process $\gamma \gamma \rightarrow \tau^{+} \tau^{-}$at the CLIC indicate the improved sensitivity on anomalous electromagnetic dipole moments of tau-lepton with respect to the existing experimental bounds by two orders of magnitude. The best sensitivities obtained on $a_{\tau}$ and $d_{\tau}$ are $-0.00012 \leq a_{\tau} \leq 0.00014$ and $\left|d_{\tau}(\mathrm{ecm})\right|=7.445 \times 10^{-19}[6]$. 


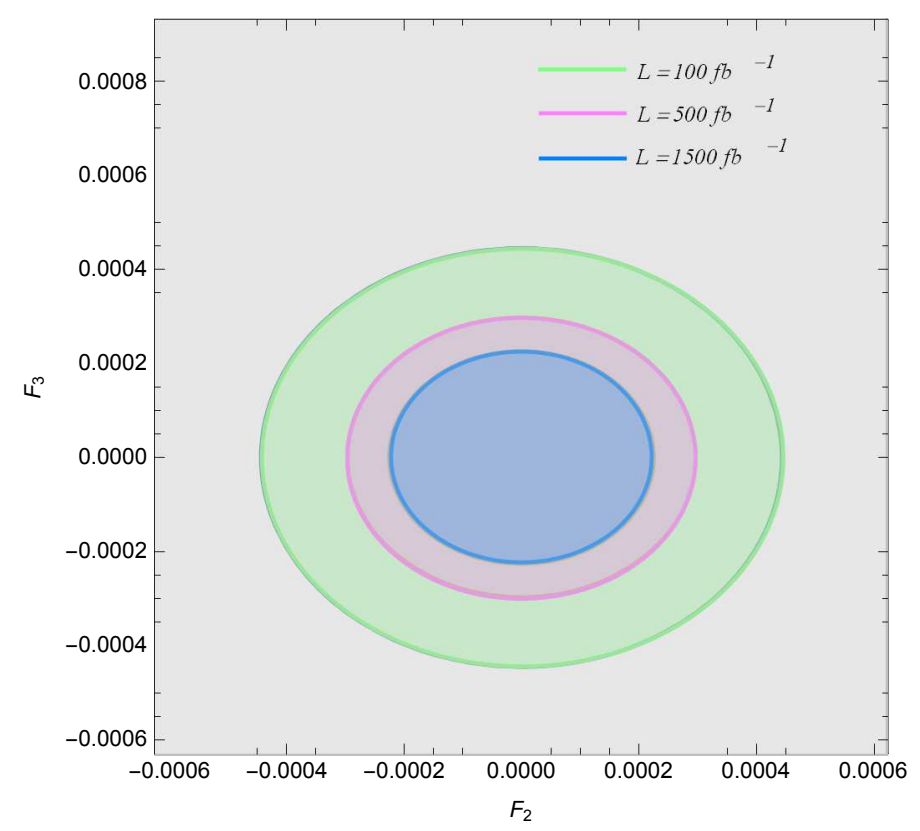

Figure 6: The same as in Figure 5, now for $\mathcal{L}=100,500,1500 \mathrm{bf}^{-1}$ and for centerof-mass energy of $\sqrt{s}=1500 \mathrm{GeV}$.

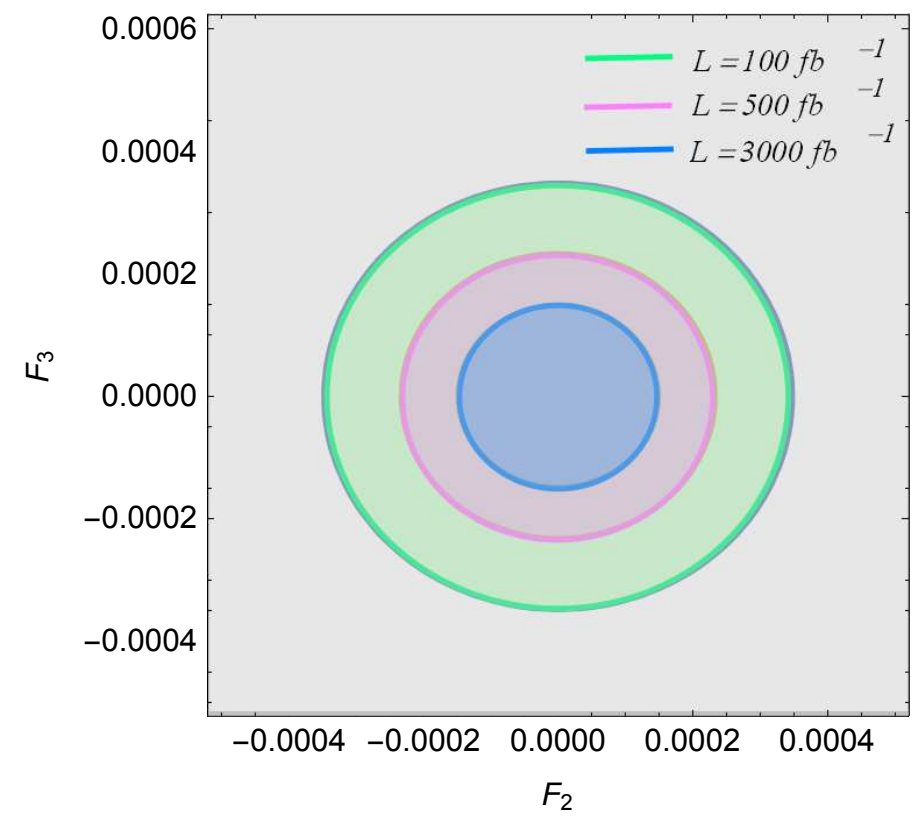

Figure 7: The same as in Figure 6, now for $\mathcal{L}=100,500,3000 \mathrm{bf}^{-1}$ and for centerof-mass energy of $\sqrt{s}=3000 \mathrm{GeV}$.

Furthermore, there has been extensive theoretical work done in new physics beyond de Standard Model that contributes to dipole moments of tau-lepton: Left-right symmetric model [17], $E_{6}$ superstring models [18], simplest little Higgs model [19], and 331 model [20]. Other limits on the MM and EDM of the $\tau$-lepton are reported in Refs. [12-14,21-25]. 


\section{Conclusion}

In conclusion, we have shown that the $\gamma \gamma \rightarrow \tau^{+} \tau^{-}$process at the CLIC leads to an improvement in the existing sensitivity estimates on the $a_{\tau}$ and $d_{\tau}$. We present an optimistic scenario regarding the potencial precision, energy, and luminosity that may be achievable at the future $e^{+} e^{-}$colliders. Our results for the process $\gamma \gamma \rightarrow \tau^{+} \tau^{-}$at the CLIC could improve the sensitivity on anomalous electromagnetic dipole moments of $\tau$-lepton with respect to the existing experimental bounds (see Table I in Ref. [6]) by 2 orders of magnitude. The best sensitivities obtained on $\tilde{a}_{\tau}$ and $\tilde{d}_{\tau}$ were $-0.00015 \leq \tilde{a}_{\tau} \leq 0.00017$ and $\left|\tilde{d}_{\tau}\right|=9.040 \times 10^{-19}$, respectively, as shown in Tables III-V in Ref. [6]. These are compared with experimental results of earlier studies for a linear collider as published by the DELPHI and BELLE Collaborations $[1,4]$.

\section{Acknowledgements}

M. A. H. R. and A. G. R. acknowledges support from SNI and PROFOCIE (México).

\section{References}

[1] J. Abdallah et al. [DELPHI Collaboration], Study of tau-pair production in photon-photon collisions at LEP and limits on the anomalous electromagnetic moments of the tau lepton, Eur. Phys. J. C 35, 159 (2004), doi:10.1140/epjc/s2004-01852-y, [arXiv:hepex/0406010].

[2] M. Acciarri et al. [L3 Collaboration], Measurement of the anomalous magnetic and electric dipole moments of the tau lepton, Phys. Lett. B 434, 169 (1998), doi:10.1016/S03702693(98)00736-9.

[3] K. Ackerstaff et al. [OPAL Collaboration], An upper limit on the anomalous magnetic moment of the tau lepton, Phys. Lett. B 431, 188 (1998), doi:10.1016/S03702693(98)00520-6, [arXiv:hep-ex/9803020].

[4] K. Inami, et al., [BELLE Collaboration], Phys. Lett. B 551, 16 (2003), doi:10.1016/S0370-2693(02)02984-2.

[5] H. Albrecht et al. [ARGUS Collaboration], A search for the electric dipole moment of the tau lepton, Phys. Lett. B 485, 37 (2000), doi:10.1016/S0370-2693(00)00630-4.

[6] M. Köksal, A. A. Billur, A. Gutiérrez-Rodríguez and M. A. Hernández-Ruíz, Modelindependent sensitivity estimates for the electromagnetic dipole moments of the $\tau$-lepton at the CLIC, Phys. Rev. D 98, 015017 (2018), doi:10.1103/PhysRevD.98.015017.

[7] S. Eidelman and M. Passera, Theory of the $\tau$ lepton anomalous magnetic moment, Mod. Phys. Lett. A 22, 159 (2007), doi:10.1142/S0217732307022694, [arXiv:hep$\mathrm{ph} / 0701260]$.

[8] J. A. Grifols and A. Mendez, Electromagnetic properties of the tau lepton from $Z^{0}$ decay, Phys. Lett. B 255, 611 (1991), doi:10.1016/0370-2693(91)90276-V, [Erratum: Phys. Lett. B 259, 512 (1991)].

[9] R. Escribano and E. Massó, Improved bounds on the electromagnetic dipole moments of the $\tau$ lepton, Phys. Lett. B 395, 369 (1997), doi:10.1016/S0370-2693(97)00059-2, [arXiv:hep-ph/9609423]. 
[10] C. Giunti and A. Studenikin, Neutrino electromagnetic interactions: a window to new physics, Rev. Mod. Phys. 87, 531 (2015), doi:10.1103/RevModPhys.87.531, [arXiv:1403.6344].

[11] A. Belyaev, N. D. Christensen and A. Pukhov, CalcHEP 3.4 for collider physics within and beyond the Standard Model, Comput. Phys. Commun. 184, 1729 (2013), doi:10.1016/j.cpc.2013.01.014, [arXiv:1207.6082].

[12] M. Köksal, S. C. İnan, A. A. Billur, Y. Özgüven and M. K. Bahar, Analysis of the anomalous electromagnetic moments of the tau lepton in $\gamma p$ collisions at the LHC, Phys. Lett. B 783, 375 (2018), doi:10.1016/j.physletb.2018.07.018, [arXiv:1711.02405].

[13] Y. Özgüven, S. C. İnan, A. A. Billur, M. Köksal and M. K. Bahar, Search for the anomalous electromagnetic moments of tau lepton through electron-photon scattering at CLIC, Nucl. Phys. B 923, 475 (2017), doi:10.1016/j.nuclphysb.2017.08.008, [arXiv:1609.08348].

[14] A. A. Billur and M. Köksal, Probe of the electromagnetic moments of the tau lepton in gamma-gamma collisions at the CLIC, Phys. Rev. D 89, 037301 (2014), doi:10.1103/PhysRevD.89.037301, [arXiv:1306.5620].

[15] I. Şahin and M. Köksal, Search for electromagnetic properties of the neutrinos at the LHC, J. High Energy Phys. 03, 100 (2011), doi:10.1007/JHEP03(2011)100, [arXiv:1010.3434].

[16] P. Achard et al. [L3 Collaboration], Muon pair and tau pair production in two photon collisions at LEP, Phys. Lett. B 585, 53 (2004) doi:10.1016/j.physletb.2004.02.012, [arXiv:hep-ex/0402037].

[17] A. Gutiérrez-Rodríguez, L. N. Luis-Noriega and M. A. Hernández-Ruíz, Limits on the dipole moments of the tau lepton via the process $e^{+} e^{-} \rightarrow \tau^{+} \tau^{-} \gamma$ in a left right symmetric model in a left right symmetric model, Mod. Phys. Lett. A 19, 2227 (2004), doi:10.1142/S0217732304014689, [arXiv:hep-ph/0403237].

[18] A. Gutiérrez-Rodríguez, M. A. Hernández-Ruíz and M. A. Pérez, Limits on the electromagnetic and weak dipole moments of the tau-lepton in $E_{6}$ superstring models, Int. J. Mod. Phys. A 22, 3493 (2007), doi:10.1142/S0217751X07036865, [arXiv:hep-ph/0611235].

[19] A. Gutiérrez-Rodríguez, Bounding the electromagnetic and weak dipole moments of the tau-lepton in a simplest little Higgs model, Mod. Phys. Lett. A 25, 703 (2010), doi:10.1142/S0217732310032238, [arXiv:0910.4217].

[20] A. Gutiérrez-Rodríguez, M. A. Hernández-Ruíz and C. P. Castañeda-Almanza, Dipole moments of the tau-lepton and Z-Z' mixing angle induced in a 331 model, J. Phys. G 40, 035001 (2013), doi:10.1088/0954-3899/40/3/035001.

[21] S. Eidelman, D. Epifanov, M. Fael, L. Mercolli and M. Passera, $\tau$ dipole moments via radiative leptonic $\tau$ decays, J. High Energy Phys. 03, 140 (2016), doi:10.1007/JHEP03(2016)140, [arXiv:1601.07987].

[22] A. Gutiérrez-Rodríguez, M. A. Hernández-Ruíz and L. N. Luis-Noriega, Magnetic moment and electric dipole moment of the $\tau$-lepton, J. Phys. Conf. Ser. 37, 25 (2006), doi:10.1088/1742-6596/37/1/006.

[23] M. Passera, Electron, muon and tau magnetic moments: A theoretical update, Nucl. Phys. Proc. Suppl. 169, 213 (2007), doi:10.1016/j.nuclphysbps.2007.03.001, [arXiv:hep$\mathrm{ph} / 0702027]$. 
[24] M. Passera, Precise mass-dependent QED contributions to leptonic $g-2$ at order $\alpha^{2}$ and $\alpha^{3}$, Phys. Rev. D 75, 013002 (2007), doi:10.1103/PhysRevD.75.013002.

[25] A. Gutiérrez-Rodríguez, M. A. Hernández-Ruíz, C. P. Castañeda-Almanza, A. EspinozaGarrido and A. Chubykalo, Limits on the electromagnetic and weak dipole moments of the tau-lepton in a 331 model, Nucl. Phys. B Proc. Suppl. 253-255, 202 (2014), doi:10.1016/j.nuclphysbps.2014.09.049, 